\title{
Developing Logistics Network from a Resilience Perspective
}

\author{
C.Y. LAM \\ Graduate School of Engineering, Hiroshima University, Japan
}

\begin{abstract}
Logistics entities are interrelated to each other, any disruption or sudden unavailability of the entities may cause severe cascading disruption effects on the functioning of the entire logistics network. In analyze the cascading disruption effects, it is important to actively concern the normal logistics practices, rather than passively emphasize on learning from the past incidents. This paper proposes a resilience analysis framework for the analysis and design of logistics network on how to help the network to cope with complexity under disruption. Under the resilience analysis framework, the logistics network is modeled as a probabilistic network graph, the resilience can then be analyzed by the reliability of all the independent connection paths among the logistics entities with connection weightings. The design of the logistics network is then further proposed by means of dependency routing. Therefore, this paper can provide an efficient way to analyze and design the stability of the logistics network as well as to understand the ability of the network to return to a stable state after disruptions from failure.
\end{abstract}

\section{Introduction}

Logistics network is one of the critical infrastructure networks that comprising identifiable assets in providing a reliable flow of products and services for the smooth functioning of a society, and it is also essential to the economics of a society [1]. In a logistics network, its entities are separated by distance and time while connections are formed among them, such that the products and services from different markets or regions can be linked together for proper functioning of a logistics network [2]. Since the entities in the logistics network are interdependent, any disruption or sudden unavailability of the entities may cause severe cascading disruption on the functioning of the entire network. A disruption (e.g. delay in a production process, traffic congestion, delivery tardiness, etc.) on an entity in the network may not only affect itself, it may also cause cascading disruption effect in the next entity of the logistics network. Moreover, the logistics network may also be disrupted by some other interrelated disruptions. For example, a flooding and rainfall may cause extensive landslides that may affect a logistics route; a power failure and blackout may cause the traffic light systems to breakdown, and it may result in heavy congestion in rush hour.

The analysis of logistics network from a risk management perspective has been conducted by many researchers, and the review can be found in [3], [4], [5]. Apart from the risk management perspective, this research study proposes a resilience analysis framework for the analysis and design of a logistics network. Resilience is a field for understanding and management of safety that focuses on how to help a network or system to cope with complexity under pressure to achieve success [6], and it has been successfully applied in various research areas [7], [8], such as ecosystems, computer networks, soci-economic systems, etc. Therefore, by adopting a resilience perspective, it can provide an efficient way to analyze the security and stability of the logistics network as well as to understand the ability of the network to return to a stable state after disruptions from failure.

\section{Logistics Network Resilience}

\subsection{Resilient Network Analysis}

Under the resilience analysis framework, a network is considered as resilient if it is impervious and resilient to disruptions, i.e. a vulnerable logistics network might be hit by a damaging disruption event, but will soon recover if it is resilient. A logistics network that is not resilient would take longer time to recover, and one that is not vulnerable would not be affected by the disruption in the first place.

In the approach of resilience analysis, the logistics network is modeled as a probabilistic graph $G(V, E)$ with a set $V=\left\{v_{i} \mid 1 \leq i \leq n\right\}$ of vertices with $n$ number of nodes and a set $E=\left\{e_{j} \mid 1 \leq j \leq\right.$ $m\}$ of $m$ number of connections between the nodes in the network $G$. Because the survivability of the logistics network depends on the number of independent connection paths between the nodes in the network [9], the resilience $r$ of a node $i$ can then be determined by the reliability $q$ of all the independent connection paths towards that node in terms of the weighting $w$ on the connection, i.e. $r_{i}=\sum_{j>1}^{n}\left(w_{i j} \times q_{i j}\right)$. Moreover, the resilience $R$ of the entire network can then be determined by the weighted sum of all the node resilience $r_{i}$, i.e. $R=\sum_{i-1}^{n}\left(w_{i} \times r_{i}\right)$. Additionally, the effect of 
redundancy can be applied for a more comprehensive resilient analysis on the logistics network, in which redundancy is regarded as a set of additional connection paths (redundant paths) that may not be significant in the normal functioning of the network, but it may be significant to the network when there are disruptions in the network. Therefore, redundancy is defined as $P(F)=\prod_{1}^{k}\left(1.0-p_{i}\right)$, where $p_{i}$ is the probability associated with each risk $k$ in the network, such that for the network facing various or increased number of risks, $P(F)$ tends toward $0 \%$ or completely secure as the number of redundancy increase, such that the hypothesis are:

- $H_{0}: \sigma_{1}^{2}=\sigma_{2}^{2}$, the variance in the resilience of the network without redundancy $\left(\sigma_{1}^{2}\right)$ is the same as the resilience of the network with redundancy $\left(\sigma_{2}^{2}\right)$.

- $H_{1}: \sigma_{1}^{2} \neq \sigma_{2}^{2}$, variance is not equal.

\subsection{Resilient Network Design}

The ability of the network in coping with disruption can be quantitatively determined by the resilience analysis framework. In knowing the resilient status of the network, it is necessary to create a robust logistics network. Based on the effect of redundancy in the network resilience analysis, it can help to further efficiently and effectively optimize the logistics network, i.e. efficiently design a route that can maximize the overall reliability of the logistics network, effectively consolidate various routes to reduce some highly overlapped routes, and enhance the integration of the logistics network. In general, the optimization approach of the logistics network is proposed to be at the restoration and recovery of the network by means of dependency routing.

The structure of a logistics network may be distorted after a disruption, the functioning of the network may only depend on its existing and usable resources, such as those still workable connection paths/ nodes, etc., so a dependency routing approach can be adopted to restore and recover the services in the network. The dependency routing approach considers the workable dependency between the entities in the network and their priorities according to the overall recovery efforts, the preferential paths in the network during disruption can then be determined and used as the restoration and recovery path. Under the dependency routing modeling approach for the restoration and recovery of the logistics network, different combinations of the network structure under different disruption scenarios are determined, such that it is useful for policy makers or management teams to develop contingency plan for the network disruption. The conceptual framework for the restoration and recovery of the logistics network by means of dependency routing is illustrated in Fig.1.

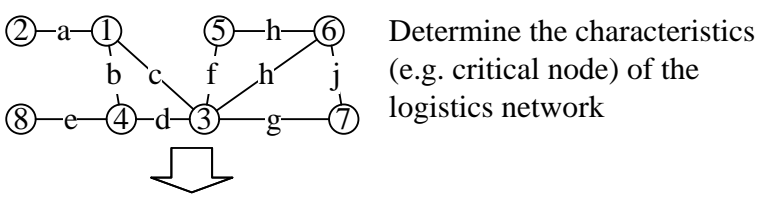

\begin{tabular}{|c|c|c|}
\hline \multicolumn{2}{|c|}{ Dependencies related to Critical Node 3: } & Determine the \\
\hline Forward Approach & Backward Approach & \\
\hline (3) $-c+(1)-a+(2)$ & (2) $-a+(1)-c+(3)$ & routings of th \\
\hline (3) $-c+$ (1)-b-(4)-e+(8) & (8)-e+(4)-b+(1)-c+(3) & characteristics \\
\hline $\begin{array}{c}\text { (3) }-f \rightarrow(5)-h \rightarrow(6)-j \rightarrow(1) \\
\vdots \\
\vdots\end{array}$ & $\begin{array}{c}\text { (1) }-\mathrm{j} \rightarrow(\text { (6) }-\mathrm{h} \rightarrow(5)-\mathrm{f} \rightarrow(3) \\
\vdots \\
\vdots\end{array}$ & $\begin{array}{l}\text { of the logistic } \\
\text { network }\end{array}$ \\
\hline
\end{tabular}

Optimization on the dependency routings of Determine the the forward / backward sequencing for the compensated network restoration and recovery of the networks logistics network after disruption

Figure 1. The conceptual framework for the restoration and recovery of the logistics network

\section{An Illustrative Example}

A logistics network with 14 nodes and 20 connections are used to illustrated the resilience analysis approach, and the single line diagram of the logistics network is presented in Fig.2, in which node 1 and node 2 are the major supply node in the network, and the reliability for the nodes and the connections are set as 0.98 and 0.88 , respectively. The data for the node weightings and the reliability of the connections are summarized in Table 1.

By adopting the resilience analysis approach, all the connections between a pair of nodes are determined by Dijkstra's Shortest Path Algorithm, and the determined shortest paths between the supply nodes and other demand nodes in the network as well as the corresponding reliability are presented in Table 2. Moreover, the resilience of the demand nodes is also determined according to the mentioned approach, and the results are presented in Table 3.

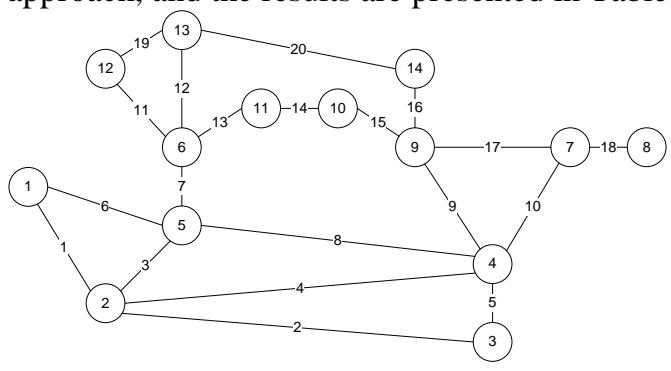

Figure 2. A logistics network with 14 nodes and 20 connections 
Table 1. Weighting of nodes

\begin{tabular}{l|lllllll}
\hline Node $i$ & 1 & 2 & 3 & 4 & 5 & 6 & 7 \\
Weight & 1.00 & 1.00 & 0.39 & 0.20 & 0.03 & 0.04 & 0.00 \\
\hline Node $i$ & 8 & 9 & 10 & 11 & 12 & 13 & 14 \\
Weight & 0.00 & 0.12 & 0.03 & 0.01 & 0.02 & 0.05 & 0.06 \\
\hline
\end{tabular}

Table 2. Reliability of Connections

\begin{tabular}{|c|c|c|}
\hline Node & Connection & Reliability \\
\hline \multirow[t]{2}{*}{3} & $(1-2) ;(6-8-5)$ & 0.928 \\
\hline & (2); (4-5) & 0.973 \\
\hline \multirow[t]{2}{*}{4} & $(1-4) ;(6-8)$ & 0.949 \\
\hline & (4); (2-5); (3-8); (1-6-7-13-14-15-9) & 0.996 \\
\hline \multirow[t]{2}{*}{5} & $(6) ;(1-3)$ & 0.973 \\
\hline & (3); (1-6); (4-8); (2-5-9-15-14-13-7) & 0.996 \\
\hline \multirow[t]{2}{*}{6} & $(6-7) ;(1-4-9-15-14-13)$ & 0.879 \\
\hline & $\begin{array}{l}(3-7) ;(4-9-15-14-13) ; \\
(2-5-10-17-16-20-12)\end{array}$ & 0.937 \\
\hline \multirow[t]{2}{*}{7} & $(1-4-10) ;(6-8-9-17)$ & 0.872 \\
\hline & $(4-10) ;(2-5-9-17)$ & 0.910 \\
\hline \multirow[t]{2}{*}{8} & $(1-4-10-18)$ & 0.600 \\
\hline & $(4-10-18)$ & 0.681 \\
\hline \multirow[t]{2}{*}{9} & $(1-4-9) ;(6-8-10-17)$ & 0.872 \\
\hline & $(4-9) ;(2-5-10-17) ;(3-7-13-14-15)$ & 0.957 \\
\hline \multirow[t]{2}{*}{10} & $(1-4-9-15) ;(6-7-13-14)$ & 0.840 \\
\hline & $(4-9-15) ;(3-7-13-14)$ & 0.872 \\
\hline \multirow[t]{2}{*}{11} & $(1-4-9-15-14) ;(6-7-13)$ & 0.850 \\
\hline & $(3-7-13) ;(4-9-15-14)$ & 0.872 \\
\hline \multirow[t]{2}{*}{12} & $(6-7-11) ;(1-4-9-16-20-19)$ & 0.830 \\
\hline & $(3-7-11) ;(4-9-16-20-19)$ & 0.850 \\
\hline \multirow[t]{2}{*}{13} & $(6-7-12) ;(1-4-9-16-20)$ & 0.850 \\
\hline & $\begin{array}{l}(3-7-12) ;(4-9-16-20) ; \\
(2-5-10-17-15-14-13-11-19)\end{array}$ & 0.913 \\
\hline \multirow[t]{2}{*}{14} & $(1-4-9-16) ;(6-7-12-20)$ & 0.840 \\
\hline & $(4-9-16) ;(3-7-12-20)$ & 0.872 \\
\hline
\end{tabular}

Table 3. Resilience of the Demand Nodes

\begin{tabular}{l|llllll}
\hline Node $i$ & 3 & 4 & 5 & 6 & 7 & 8 \\
Resilience $r_{i}$ & 0.98 & 1.90 & 1.93 & 1.78 & 1.74 & 1.25 \\
\hline Node $i$ & 9 & 10 & 11 & 12 & 13 & 14 \\
Resilience $r_{i}$ & 1.79 & 1.67 & 1.68 & 1.64 & 1.72 & 1.67 \\
\hline
\end{tabular}

In Table 3, it can be found that the resilience of the demand nodes are ranging from 0.98 to 1.93 , and the associated resilience index of the entire logistics network is 1.480 , in which a higher resilience index representing a higher reliability of the network as well as its higher ability to return to its normal operating state after disruptions.

In order to determine the effect of redundancy in network structure, five redundant connections are added incrementally to the logistics network so as to increase the average degree of the nodes to a value of above 3 , in which the redundant connections are added between node 1 and 12 (connection 21), node 11 and 13 (connection 22), node 10 and 14 (connection 23), node 8 and 14 (connection 24), and node 3 and 8 (connection 25). The network diagram with 5 additional redundant connections is shown in Fig.3, in which the average degree of the nodes increases as the number of redundant connections increases (from 2.8 to 3.5). Additionally, the matrixes for the number of paths between a pair of nodes in the network are presented in Table IV, in which the number of paths increases as the number of redundant connections increases, such that the number of alternative paths for the supply to reach the demand nodes is increased.

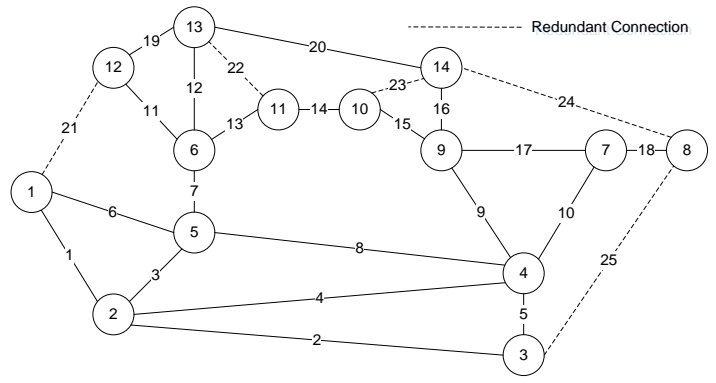

Figure 3. A logistics network with 5 additional redundant connections

Table 4. Matrix for the Number of Connections

\begin{tabular}{|c|c|c|c|c|c|c|c|c|c|c|}
\hline & \multicolumn{2}{|c|}{ 1* } & \multicolumn{2}{|c|}{ 2* } & \multicolumn{2}{|c|}{$3^{*}$} & \multicolumn{2}{|c|}{$4 *$} & \multicolumn{2}{|c|}{$5^{*}$} \\
\hline $\mathrm{n}$ & 1 & 2 & 1 & 2 & 1 & 2 & 1 & 2 & 1 & 2 \\
\hline 3 & 2 & 2 & 2 & 2 & 2 & 2 & 2 & 2 & 3 & 3 \\
\hline 4 & 3 & 4 & 3 & 4 & 3 & 4 & 3 & 4 & 3 & 4 \\
\hline 5 & 3 & 4 & 3 & 4 & 3 & 4 & 3 & 4 & 3 & 4 \\
\hline 6 & 3 & 4 & 3 & 4 & 3 & 4 & 3 & 4 & 3 & 4 \\
\hline 7 & 2 & 2 & 2 & 2 & 2 & 2 & 3 & 3 & 3 & 3 \\
\hline 8 & 1 & 1 & 1 & 1 & 1 & 1 & 2 & 2 & 3 & 3 \\
\hline 9 & 3 & 4 & 3 & 4 & 3 & 4 & 3 & 4 & 3 & 4 \\
\hline 10 & 2 & 2 & 2 & 2 & 3 & 3 & 3 & 3 & 3 & 3 \\
\hline 11 & 2 & 2 & 3 & 3 & 3 & 3 & 3 & 3 & 3 & 3 \\
\hline 12 & 3 & 3 & 3 & 3 & 3 & 3 & 3 & 3 & 3 & 3 \\
\hline 13 & 3 & 3 & 3 & 4 & 3 & 4 & 3 & 4 & 3 & 4 \\
\hline 14 & 2 & 2 & 2 & 2 & 3 & 3 & 3 & 4 & 3 & 4 \\
\hline
\end{tabular}

As the redundant connections are added incrementally to the logistics network, the resilience of the demand nodes and the entire network can then be determined, and the results and their plots are presented in Table V-VI and Figures 4-5, respectively.

Table 5. Resilience of the Demand Nodes

\begin{tabular}{c|c|c|c|c|c}
\hline $\mathrm{n}$ & $r_{i}=1$ & $r_{i}=2$ & $r_{i}=3$ & $r_{i}=4$ & $r_{i}=5$ \\
\hline 3 & 1.863 & 1.863 & 1.863 & 1.863 & 1.914 \\
4 & 1.930 & 1.933 & 1.933 & 1.933 & 1.933 \\
5 & 1.948 & 1.948 & 1.948 & 1.948 & 1.951 \\
6 & 1.914 & 1.914 & 1.914 & 1.914 & 1.918 \\
7 & 1.747 & 1.747 & 1.747 & 1.854 & 1.873 \\
8 & 1.256 & 1.256 & 1.256 & 1.656 & 1.877 \\
9 & 1.890 & 1.890 & 1.890 & 1.890 & 1.890 \\
10 & 1.678 & 1.678 & 1.838 & 1.838 & 1.847 \\
11 & 1.668 & 1.863 & 1.863 & 1.863 & 1.863 \\
12 & 1.907 & 1.907 & 1.907 & 1.907 & 1.907 \\
13 & 1.887 & 1.903 & 1.903 & 1.908 & 1.908 \\
14 & 1.710 & 1.710 & 1.834 & 1.869 & 1.889 \\
\hline
\end{tabular}


The results and the associated plotting show that the resilience of the network increases as the number of redundant connections increases, which means that the ability of the network to cope with disruption is increased.

Table 6. Resilience Index for the Network With Different Redundancy

\begin{tabular}{cccccc}
\hline Redundancy & 1 & 2 & 3 & 4 & 5 \\
\hline$R$ & 1.865 & 1.869 & 1.883 & 1.888 & 1.911 \\
\hline
\end{tabular}

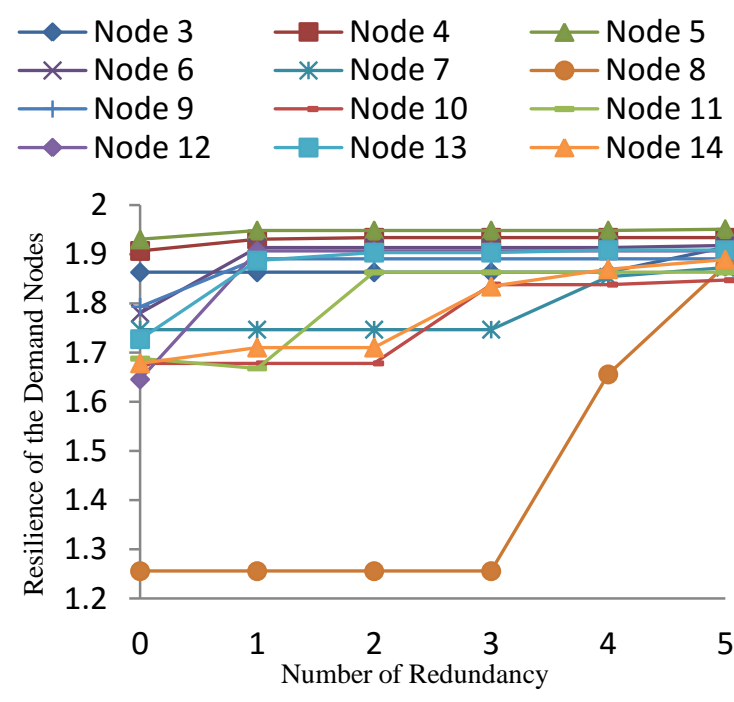

Figure 4. Changes in the resilience of the demand nodes

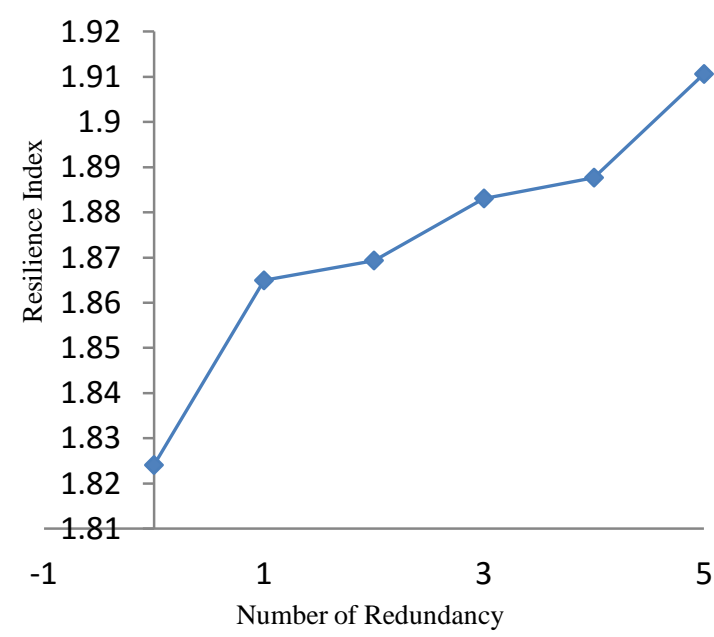

Figure 5. Changes in the resilience index of the entire network

\section{Conclusion}

Resilience is an emerging safety management paradigm concerned with normal practice, rather than emphasizing learning from incidents. In this paper, a resilience framework is proposed for the analysis and design of logistics network. The logistics network is firstly modeled as a probabilistic graph $G(V, E)$ with a set of vertices with $n$ number of nodes and a set of edges with $m$ number of connections between the nodes in the network, such that the dependencies of the nodes in the logistics network can be identified, and the resilience of the logistics network can also be analyzed by the reliability of all the independent connection paths between pair of nodes with some weighting factors. By knowing the resilience and the effect of redundancy in logistics network, an efficient and effective logistics network can then be designed by maximizing the overall reliability of the logistics network in terms of dependency routing. The proposed research study can also be applied for investigating resilience at any node within the network, such that a more resilient logistics network can be developed. The further work of this research will be on the integration of the framework in the dynamic modeling, analysis and design of the resilience of the logistics networks.

\section{References}

[1] W.J. Clinton, "Executive order 13010 - Critical infrastructure protection", Federal Register, Vol.61(138), pp.37347-37350, 1996.

[2] C.Y. Lam and K. Tai, "Evaluating the Reliability of Infrastructure Networks by Resilience Analysis", Proceedings of the 2012 IEEE IEEM, pp.1165-1169, 2012.

[3] C. Busse and C.M. Wallenburg, "Innovation management of logistics service providers: foundations, review, and research agenda", International Journal of Physical Distribution \& Logistics Management, Vol.41(2), pp.187-218, 2011.

[4] H. Jafari, " Logistics flexibility: A systematic review", International Journal of Productivity and Performance Management, Vol.64(7), pp.947-970, 2016.

[5] N. Anand, R. van Duin, H. Quak, and L. Tavasszy, "Relevance of City Logistics Modelling Efforts: A Review", Transport Reviews, Vol.35(6), pp.701-719, 2015.

[6] E. Hollnagel, D. D. Woods, and N. Leveson, "Resilience Engineering: Concepts and Precepts", Ashgate Publishing, Aldershot, UK, 2006.

[7] S.E. Birkie, P. Trucco, and M. Kaulio, "State-of-theArt Review on Operational Resilience: Concept, Scope and Gaps", Advances in Production Management Systems. Competitive Manufacturing for Innovative Products and Services, Vol.398 of the series IFIP Advances in Information and Communication Technology, pp.273-280, 2012.

[8] A. Sharifi, "A critical review of selected tools for assessing community resilience", Ecological Indicators, Vol.69, pp.629-647, 2016. 
International Journal of Sustainable Energy Development (IJSED), Volume 5, Issue 2, December 2016

[9] D. Wang, and W.H. Ip, "Evaluation and Analysis of Logistics Network Resilience With Application to Aircraft Servicing". IEEE Systems Journal, Vol.3(2), pp.166-173, 2009.

[10] J. Cohen, "Statistical Power Analysis for the Behavioral Sciences", Lawrence Erlbaum Associates, Hillsdale, New Jersey, 1988. 